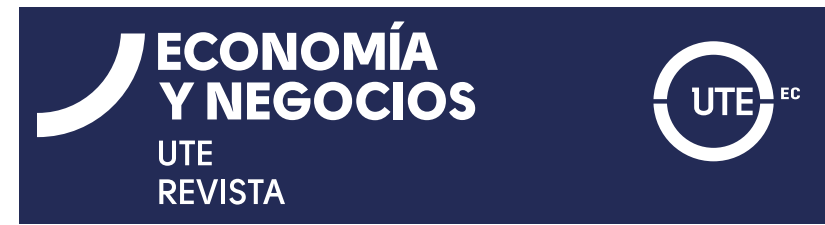

Economía y Negocios, 2021, 12(02), 32-51.

http://revistas.ute.edu.ec/index.php/economia-y-negocios

\section{ARTÍCULO DE INVESTIGACIÓN}

ISSN-e: 2602-8050

Recibido (Received): 2021/03/23 Aceptado (Accepted): 2021/10/06 Publicado (Published): 2021/12/06

https://doi.org/10.29019/eyn.v12i2.853

\title{
Cultura Organizacional y Desempeño Docente en Instituciones Públicas de Educación Superior
}

\section{Organizational Culture and Teaching Performance in Public Institutions of Higher Education}

\author{
Galo Ramiro MORENO-BASTIDAS ${ }^{1}$ (iD) y Rosario del Carmen PINEDA-LÓPEZ ${ }^{1}$ (iD
}

1 Universidad de las Fuerzas Armadas (ESPE), Departamento de Ciencias Económicas, Administrativas y de Comercio. Quito, Ecuador.

\begin{abstract}
Resumen
Por medio de esta investigación se pretendió establecer la relación entre cultura organizacional y desempeño laboral en las funciones sustantivas de docencia, investigación y vinculación con la sociedad de las universidades públicas de la provincia Pichincha (Ecuador). Se seleccionaron tres universidades: Universidad de las Fuerzas Armadas (ESPE), Escuela Politécnica Nacional (EPN) y Universidad Central del Ecuador (UCE). La muestra fue de 304 docentes. Para medir y analizar los datos se estableció una metodología basada en las características cuantitativas, cualitativas, individuales y grupales de la data existente.

Los resultados muestran que las universidades tienen una cultura organizacional en la que se desarrollan aspectos, tales como: integración, relaciones interpersonales, trabajo en equipo y comunicación. De las evaluaciones se obtuvo conclusiones similares: en general, los docentes se desempeñan adecuadamente en relación con la planificación, evaluación, contenidos, entre otros. El análisis de correlación desarrollado permitió demostrar una correlación significativa entre los valores medios de cultura organizacional y desempeño docente. De esta forma se corroboró la hipótesis acerca de que las variables de la cultura organizacional consideradas sí inciden en el desempeño laboral de los profesores universitarios.
\end{abstract}

\section{Palabras clave}

Cultura organizacional, desempeño docente, universidades públicas.

\begin{abstract}
Through this research, the aim was to establish the relationship between organizational culture and work performance in the substantive functions of teaching, research and connection with society in the public universities of the Pichincha province (Ecuador). Three universities were selected: University of the Armed Forces (ESPE), National Polytechnic School (EPN) and Central University of Ecuador (UCE). The sample was 304 teachers. To measure and analyze the data, a methodology was established based on the quantitative, qualitative, individual and group characteristics of the existing data.

The results show that universities have an organizational culture in which aspects are developed, such as: integration, interpersonal relationships, teamwork and communication. Similar conclusions were obtained from the evaluations: in general, teachers perform adequately in relation to planning, evaluation, content, among others. The correlation analysis developed allowed to demonstrate a significant correlation between the mean values of organizational culture and teaching performance. In this way, the hypothesis was corroborated that the variables of the organizational culture considered do affect the job performance of university professors.
\end{abstract}

\section{Keywords}

Organizational culture, teaching performance, public universities.

Códigos de clasificación JEL: J4, M12, M14. 


\section{Introducción}

La geopolítica, la cultura, la economía, la educación, la tecnología, la ciencia y la sociedad en general han sido protagonistas de nuevas perspectivas y enfoques dentro de un novedoso contexto: la globalización. En consecuencia, nuevas directrices orientan el quehacer de la humanidad, que también impactan en las instituciones en las cuales se forma el talento humano de cara a esta realidad cambiante. Las universidades no han sido la excepción y sus actividades sustantivas de docencia, investigación y vinculación con la sociedad asumen y responden a nuevos patrones culturales para el cumplimiento de su función.

Ante estos retos, las universidades, desde los paradigmas de la sociedad del conocimiento, del acceso a la educación superior, de la evaluación y acreditación, así como de la universidad contemporánea, deben ampliar su visión para gestionar sus recursos, renovar sus valores, creencias, comportamientos, identidad y estrategias, a fin de que la cultura resultante pueda cohesionar a todos sus miembros, especialmente a su cuerpo docente en torno a la misión institucional. Consecuentemente, las universidades deben establecer estrategias que coadyuven al fortalecimiento de una cultura organizacional, donde las creencias, valores y aspectos conductuales elevados favorezcan al desempeño de su personal académico en las funciones sustantivas (docencia, investigación y vinculación), las cuales dan vida y propósito al quehacer universitario (De Aparicio et al., 2017).

Actualmente, para que las universidades ecuatorianas públicas y privadas cumplan con los objetivos institucionales -en el marco de sus funciones primordiales- dependen del desempeño óptimo, cohesionado y efectivo de su cuerpo docente en estos quehaceres académicos. Sin embargo, aun cuando este tipo de instituciones forman profesionales a través del conocimiento, en el Ecuador existen pocas investigaciones acerca de la relación entre la cultura organizacional y el desempeño laboral del cuerpo docente, siendo esta la base para un diagnóstico y posterior toma de decisiones para lograr mejores resultados en la gestión que estas realizan.

Ante este escenario cobra valor la siguiente interrogante: ¿La cultura de las universidades públicas ecuatorianas incide en el desempeño laboral de su cuerpo docente en el cometido de las funciones de docencia, investigación y vinculación? A efectos de investigar la cultura organizacional como elemento clave en el desempeño de los profesores en las universidades públicas de la provincia de Pichincha, se parte de evaluar la incidencia de la cultura organizacional en el desempeño del personal docente en las funciones sustantivas de las instituciones públicas de educación superior ubicadas en la provincia de Pichincha en la República del Ecuador.

El objetivo radica en establecer la relación entre cultura organizacional y desempeño del personal docente en las funciones sustantivas de docencia, investigación y vinculación en las universidades públicas de la provincia de Pichincha.

\section{Revisión de la literatura}

\section{Cultura organizacional}

La cultura organizacional ha manifestado cambios en su desarrollo conceptual e importantes teóricos la han definido considerando diferentes formas y puntos de vista. Los principales aportes más recientes y destacados son los siguientes:

- Conjunto de prácticas sociales materiales e inmateriales que dan cuenta de las características que distinguen a una comunidad, ya que establecen una 
atmósfera afectiva común y un marco cognitivo compartido (Anzola, 2003).

- Es un patrón de supuestos básicos compartidos que el grupo aprende conforme resuelve problemas de adaptación externa e integración interna, los cuales funcionan suficientemente bien para ser considerados válidos y, por tanto, ser enseñados a nuevos miembros como la forma correcta de percibir, pensar y sentir en relación con esos problemas (Schein, 2004).

- Conjunto de significados y valores que los miembros de una organización tienen. Estos significados y valores son los bloques de construcción de cultura organizacional y se expresan mediante símbolos, comportamientos y estructuras (de Hilal et al., 2009).

- La identidad de los miembros, el énfasis de grupo, el perfil de la decisión, la integración, el control, la tolerancia al riesgo, los criterios de recompensa, la tolerancia al conflicto, el perfil de los fines o medios y el enfoque de la organización son parte fundamental de la cultura organizacional, debido a que esta puede facilitar o dificultar la solución de los problemas relacionados con la adaptación al entorno y la integración interna (Salazar Estrada et al., 2009).

- Patrón de supuestos básicos que se manifiestan a través del comportamiento de una organización (Cheung et al., 2012; Rivera et al., 2018).

- Es un recurso estratégico que posee el valor de asegurar la continuidad y permanencia de las organizaciones (Sampieri et al., 2014).

Para complementar, es preciso considerar lo propuesto por Reinoso y Araneda (2007), quienes afirman que la cultura incluye un conjunto de valores compartidos por la gran mayoría de los integrantes de una entidad que los diferencia de otras. Entre estos componentes constan:

- Identidad de los integrantes: si las personas se identifican más con la institución, su labor, su lugar de trabajo o su profesión.

- Énfasis de grupo: si las tareas se emprenden en grupo o si se realizan individualmente.

- Carácter de la decisión: si durante el proceso de toma de decisiones prevalecen las operaciones o el talento humano.

- Integración: si los departamentos actúan de manera organizada o independiente.

- Control: si existe una conducta orientada hacia una fuerte regulación o se encamina hacia el autocontrol.

- Tolerancia al riesgo: si se impulsa o no un estilo creativo, innovador y con iniciativa para favorecer la ejecución de las tareas, entre otros.

- Criterios de recompensa: si se sustentan en el desempeño o en la preferencia, la experiencia u otros elementos.

- Tolerancia al conflicto: si se impulsa o no la contienda funcional como factor del progreso institucional.

- Rasgo o aspecto de los fines o medios: si para la dirección institucional predominan los resultados o los medios utilizados para alcanzarlos.

- Perspectiva de la organización: si la dirección de la organización está orientada hacia el entorno externo o hacia el interior de la corporación.

\section{El desempeño docente universitario}

González-Such y Subaldo-Suizo (2015) explican que el docente universitario se desempeña en un mundo donde los cambios además de múltiples se generan a gran velocidad, tal como sucede con el avance tecnológico; por tanto, quienes comiencen 
sus estudios universitarios terminarán sus carreras en una sociedad muy diferente a la de la fecha de inicio (Gómez López \& Valdés Dávila, 2019). Los autores consultados consideran que el docente con frecuencia compagina diversos roles, en ocasiones algo contradictorias, en el marco de un equilibrio algo inseguro en distintos escenarios educativos; consecuentemente, cada vez más requerirá de la aplicación de estrategias novedosas y didácticas, lo cual genera dinámicas fundamentadas en la realidad actual.

En este contexto, el docente afronta, y con mucho más impacto a futuro, la exigencia de incluir en sus labores cotidianas novedosas y variadas fuentes de información, lo cual transforma su rol tradicional. La inclusión de las nuevas tecnologías genera cambios significativos en la didáctica del profesor actual (Montesinos Flores, 2014).

El desempeño docente es toda acción ejecutada por el docente, en respuesta a lo que le corresponde, tanto en las aulas como en la institución educativa. En el aula, el docente debe fomentar un ambiente favorable, un clima de armonía, pero también diseñar y desarrollar esquemas para la sesión de aprendizaje (aprendizajes esperados, secuencia de actividades y evaluación) tomando en cuenta los intereses y necesidades del alumnado, de tal forma que su actividad pedagógica influya significativamente en el compromiso y comportamiento estudiantil. Para ello debe promover la participación activa de los educandos y compartir con ellos el desarrollo de las clases.

Otra perspectiva para definir al desempeño docente es mediante la valoración del uso de capacidades y recursos intelectuales. Estas permitirán, por medio de estrategias metodológicas y pedagógicas, alcanzar el éxito en el aprendizaje, desarrollar las competencias estudiantiles en la búsqueda de una vida profesional plena, promover la participación en la gestión de instituciones educativas, fortalecer los valores democrá- ticos del alumnado, apoyar la formulación, ejecución y control de políticas educativas locales, regionales o nacionales (Martínez Ruiz \& Lavín García, 2017).

De acuerdo con los criterios de González-Such y Subaldo-Suizo (2015), las características del desempeño docente universitario son múltiples y variadas, pero hay consideraciones elementales que determinan un buen desempeño de la profesión en cuatro aspectos fundamentales:

- Planificación y organización para favorecer el aprendizaje del alumnado,

- Educación orientada y dirigida hacia el aprendizaje estudiantil,

- Intervención en la administración del proyecto académico institucional de la universidad en un proceso articulado con los requerimientos de la sociedad y

- Perfeccionamiento del profesionalismo e identidad del docente.

Asimismo, los autores consideran que el desempeño de la función docente se diferencia en dos particularidades fundamentales que caracterizan a una profesión:

- En primer lugar, la facultad e independencia de su ejercicio profesional está restringida por disposiciones y medidas regulatorias que procuran garantizar el derecho fundamental a una educación universal mediante una oferta académica en términos de justicia e igualdad.

- En segundo lugar, la enseñanza se aparta de la independencia de criterios y del aislamiento personal que caracteriza al resto de las profesiones, además de introducir como señal de identidad el resguardo cuidadoso de las relaciones interpersonales con los estudiantes y el contenido afectivo del propio empeño personal.

El docente, de manera individual, "debe ratificar el conocimiento, presentar el desa- 
rrollo de las nuevas ideas, invitar al estudiante a ser partícipe de la generación de nuevos saberes y realidades" (Murraina et al., 2017, p. 3).

\section{Cultura organizacional y el desempeño docente}

Las organizaciones deben comprometerse para asumir el éxito en un marco de apertura al aprendizaje, en el cual los integrantes se identifiquen con la entidad y logren obtener un desempeño laboral acorde a los requerimientos internos y externos.

Las universidades asumen como misión fundamental la formación académicoorganizacional de profesionales idóneos y transformadores de la realidad de un país mediante la implementación mancomunada y articulada de sus funciones sustantivas como la docencia, investigación y vinculación con la sociedad. Desde este punto de vista, los profesores que facilitan sus servicios docentes en instituciones universitarias ejecutan funciones muy significativas que deben ser evaluadas; no obstante, la evaluación debe ser cónsona con la filosofía universitaria (Sineases, 2017).

Para evaluar el desempeño docente deben contemplarse los valores, las creencias y los supuestos que respaldan la cultura universitaria y que facilitan el cumplimiento de estas funciones. Para ello, la organización, a la par de sus principios, debe propender a una formación adecuada que permita la mejora continua de la función pedagógica del docente. Asimismo, se deben brindar todas las facilidades para convertirlo en un investigador, aportándole las orientaciones apropiadas para su participación en proyectos de investigación científica y herramientas para divulgar resultados. De la misma manera, debe preparar todos los aspectos necesarios y precisos para su participación en proyectos que permitan su vinculación con la sociedad, la cual debe estar respaldada por la institución (Garcés, 2013; Segredo Pérez, 2016).

Para adecuar la cultura universitaria y que esta incida en las funciones sustantivas del cuerpo docente, la institución debe promover una evaluación que tienda a la autoevaluación; es decir, a la apreciación personal que tienen los docentes sobre sus avances; teniendo en cuenta, además, la evaluación realizada por los pares académicos, especialmente para determinar su participación en los aspectos relevantes de la docencia, investigación y vinculación (Loredo, 2015). Por último, se debe llevar a cabo una apropiada y justa heteroevaluación, la cual radica en la apreciación evaluativa que hace el alumnado acerca de la calidad del aprendizaje transmitido por el cuerpo docente (Moreno Bastidas, 2020). Esta forma de evaluar el desempeño docente en el marco de la cultura universitaria sirve para determinar las fases de planificación, implementación y medición de resultados de forma complementaria y más objetiva.

\section{Materiales y métodos}

El enfoque metodológico se fundamentó en una investigación mixta. Adicionalmente, la investigación, acorde con lo propuesto por Hernández et al. (2014) y Arias (2012), tiene carácter transversal, analítico, exploratorio, correlacional y de campo, porque indaga en la incidencia y en los valores en que se manifiestan los diferentes aspectos que se investigan en un momento determinado del tiempo. Este enfoque permite analizar y explicar los hechos y fenómenos tal y como se observan en la realidad, en un primer acercamiento, en la búsqueda de las causas y consecuencias.

En esta investigación se trabajó con las universidades públicas de la provincia de Pichincha. En cuanto al alcance, mediante el diseño metodológico elegido, este se limitó a las siguientes universidades: Universidad 
de las Fuerzas Armadas (ESPE), Escuela Politécnica Nacional (EPN) y Universidad Central del Ecuador (UCE). Estas universidades fueron seleccionadas con base en criterios de su importancia regional y nacional, debido a que son las tres únicas universidades públicas con clasificación A y B. De acuerdo con el Consejo de Aseguramiento de la Calidad de la Educación Superior (CACES, 2018, 2019), a nivel del Ecuador, la UCE está en categoría $B$, las demás, en la $A$.

\section{Técnicas de observación utilizadas}

Entrevistas no estructuradas: se entrevistó a los vicerrectores de las universidades mencionadas. Las entrevistas fueron analizadas para obtener información de las autoridades centrales de cada universidad sobre las dos variables de estudio: cultura organizacional de las universidades en estudio y desempeño laboral del docente universitario.

Encuestas: se aplicaron a los docentes de estas universidades para conocer su apreciación sobre la cultura organizacional predominante, así como una autoevaluación de su desempeño.

Informes de evaluación sobre el desempeño docente en las universidades: se utilizó como fuente de información secundaria los informes de evaluación del desempeño docente de los que disponen las universidades públicas. Esos informes se elaboran respetando la normativa ecuatoriana y en coordinación con la Unidad de Acreditación y Aseguramiento de la Calidad (UAACES).

\section{Variables de investigación}

Los elementos de la cultura organizacional y del desempeño docente en las funciones sustantivas de estas universidades públicas permiten determinar cuáles factores de la cultura organizacional inciden directamente en el desempeño docente y funcional de estas instituciones. A continuación, se presentan las variables de investigación (tabla 1).

Tabla 1. Análisis de las variables de investigación

\begin{tabular}{|c|l|l|}
\hline $\begin{array}{c}\text { Variables } \\
\text { seleccionadas }\end{array}$ & \multicolumn{1}{|c|}{ Autores } & \multicolumn{1}{c|}{ Preguntas de la investigación } \\
\hline Comunicación & $\begin{array}{l}\text { (Greenbaum, 2017; Lud- } \\
\text { low \& Panton, 1997) }\end{array}$ & $\begin{array}{l}\text { ¿Existe una buena comunicación entre los compañeros de trabajo? } \\
\text { ¿El jefe directo comunica si se está realizando buen o mal trabajo? }\end{array}$ \\
\hline Trabajo en equipo & $\begin{array}{l}\text { (Mascaray, 1998) } \\
\text { (Sánchez \& Otálora, }\end{array}$ & $\begin{array}{l}\text { ¿Los docentes de otras aulas y/o áreas se ayudan cuando es } \\
\text { necesario? } \\
\text { ¿Las reuniones de coordinación con los miembros de otras áreas } \\
\text { son frecuentes? }\end{array}$ \\
\hline interpersonales & $\begin{array}{l}\text { (Daft, 2011; Montes Pa- } \\
\text { dilla, 2016) }\end{array}$ & $\begin{array}{l}\text { ¿Se mantienen buenas relaciones con los miembros de la } \\
\text { organización? } \\
\text { ¿Se recibe buen trato por parte de los compañeros de trabajo? }\end{array}$ \\
\hline Integración & (Loren, 2012) & $\begin{array}{l}\text { ¿Se celebran fechas especiales? } \\
\text { ¿Se utilizan sistemas de incentivos para premiar la labor docente? } \\
\text { ¿La institución y los trabajadores se identifican con el logo y los } \\
\text { colores corporativos? }\end{array}$ \\
\hline Contenidos & (Figueroa, 2016) & $\begin{array}{l}\text { ¿Realiza la diversificación de los contenidos teniendo en cuenta } \\
\text { la realidad? } \\
\text { Para la diversificación ¿usa como base las nuevas tendencias en } \\
\text { materia de educación superior? }\end{array}$ \\
\hline
\end{tabular}




\begin{tabular}{|c|l|l|}
\hline Planificación & (Góngora et al., 2014) & $\begin{array}{l}\text { ¿Los objetivos estratégicos guardan relación con la visión y misión } \\
\text { de la institución? } \\
\text { ¿Se elabora la planificación de aula y/o área? }\end{array}$ \\
\hline Evaluación & (Linares et al., 2013) & $\begin{array}{l}\text { ¿La evaluación de desempeño motiva para aplicar nuevas metodo- } \\
\text { logías y realizar mejor trabajo? } \\
\text { ¿Se debe realizar con frecuencia la autoevaluación del desempe- } \\
\text { ño docente? }\end{array}$ \\
\hline $\begin{array}{c}\text { nesempeño } \\
\text { docente }\end{array}$ & (Benítez Torres, 2007) & $\begin{array}{l}\text { ¿Organiza, planifica y ejecuta el trabajo universitario para el desa- } \\
\text { rrollo de las áreas? } \\
\text { ¿Participa en reuniones metodológicas y de orientación para el } \\
\text { cumplimiento de las actividades previstas en las áreas? }\end{array}$ \\
\hline
\end{tabular}

\section{Evaluación de la validez del instrumento}

El cuestionario fue diseñado por los autores considerando las variables de la cultura organizacional y analizando herramientas validadas de diferentes autores, con el fin de obtener una encuesta propia aplicada al caso, en la que se pueda establecer las variables por estudiar y las características que puedan ser compiladas e identificadas sobre cultura organizacional. Para medir la fiabilidad del cuestionario se calculó el alpha de Cronbach con el programa estadístico SPSS (Statiscal Package for the Social Sciences).

De acuerdo con el alpha de Cronbach, el instrumento utilizado para medir la cultura organizacional e integración es confiable, debido a que se constató que las dimensiones de cultura organizacional e integración superaron el valor de 9; lo que demostró que es excelente como instrumento de me- dición. La dimensión trabajo en equipo se ubicó en un valor superior a 8, lo que se interpreta como un buen instrumento. Por último, la dimensión comunicación se ubicó en un valor superior a 7, lo que significa que el instrumento es aceptable.

\section{Población y muestra}

La población corresponde a la totalidad de docentes titulares de las universidades públicas de Pichincha seleccionadas para el estudio y de acuerdo con la base de datos entregada por las instituciones de educación superior analizadas. A continuación, se determinó una muestra representativa para la aplicación de la encuesta caracterizada por docentes que participen en los procesos sustantivos descritos. Además, se utilizó la siguiente simbología para las universidades: ESPE (U1), EPN (U2) y UCE (U3) (tabla 2).

Tabla 2. Cantidad de docentes por universidad

\begin{tabular}{|l|c|}
\hline \multicolumn{1}{|c|}{ Universidad } & Cantidad de Docentes \\
\hline ESPE $^{*}$ & 500 \\
\hline EPN $^{\star *}$ & 324 \\
\hline UCE $^{\star \star \star}$ & 611 \\
\hline Total & 1435 \\
\hline
\end{tabular}

Nota: *Escuela Politécnica del Ejército (ESPE, 2019), **Escuela Politécnica Nacional (SII-EPN, 2018) y ***Universidad Central del Ecuador (CACES, 2018) 
Para obtener una muestra significativa de la población se usó la fórmula para el cálculo de muestra para datos finitos, debido a que se cuenta con el total de la población en estudio. La fórmula utilizada está establecida, de acuerdo con Valdivieso et al. (2011), para muestras finitas y con el valor P. Los valores seleccionados se presentan a continuación:

$\mathrm{N}: 1435$ docentes

Z $\alpha / 2: 1.96$ para un $95 \%$ de confianza

$P: 0.5$

e: error aceptable 0.05
El tamaño de muestra permitió obtener resultados con un $95 \%$ de confianza y un error de $+/-5 \%$, y $n$ es igual a 303.2 que se aproxima a 304 docentes. Dado el tamaño de muestra seleccionada, se hizo un muestreo aleatorio estratificado (Casal \& Mateu, 2003) considerando proporcionalmente el número de docentes, de acuerdo con la universidad para obtener una muestra significativa por cada universidad de la siguiente manera (tabla 3).

Tabla 3. Cantidad proporcional de muestra por universidad

\begin{tabular}{|l|c|c|c|}
\hline \multicolumn{1}{|c|}{ Universidad } & Cantidad de docentes & Proporción & Tamaño de muestra \\
\hline ESPE & 500 & $34.84 \%$ & 106 \\
\hline EPN & 324 & $22.58 \%$ & 69 \\
\hline UCE & 611 & $42.58 \%$ & 129 \\
\hline Total & 1435 & $100 \%$ & 304 \\
\hline
\end{tabular}

La encuesta fue aplicada mediante las plataformas digitales de las universidades. Se envió la solicitud de información directamente a los docentes, para ello se solicitó el aval de las autoridades universitarias.

\section{Metodología de análisis y medición de los resultados}

Se desarrollaron tres fases de análisis de los resultados:

a. Establecimiento de la relación individual entre los resultados de cultura.

b. Análisis de los resultados totales por universidad.

c. Relación cualitativa entre variables de estudio.

A continuación, se presenta la relación individual de resultados:

- TD (Totalmente en desacuerdo): 1 punto.
- ED (En desacuerdo): 2 puntos.

- NA/ND (Ni de acuerdo / ni en desacuerdo): 3 puntos.

- DA (De acuerdo): 4 puntos.

- TA (Totalmente de acuerdo): 5 puntos.

\section{Análisis y resultados}

\section{Caracterización general de los docentes encuestados}

Se pudo caracterizar a un total de 310 docentes de las universidades en estudio, con una frecuencia mayor para la U3 que corresponde al $41.6 \%$ porque es la universidad con mayor cantidad de docentes. Los datos de la caracterización son los siguientes:

- La edad media de los docentes de todo el estudio está mayormente entre 36 y 50 años de edad (51.3\%), y un porcentaje menor con edades menores 0 mayores a este rango. 
- La mayor parte de docentes pertenece al género masculino (70 \%).

- La formación corresponde a magister en un $73 \%$ y PhD en un $23 \%$.

- Los docentes encuestados de las tres universidades tienen un tiempo de servicio en la universidad entre 1 a 10 años para un $48 \%$, y un $29 \%$ para los que tienen de 11 a 20 años.

- La mayor parte de los encuestados tiene nombramiento laboral (84 \%) y un $15 \%$ son docentes a contrato.

- El tiempo de dedicación que tienen en cada universidad es de tiempo completo en un $87 \%$, y solamente a tiempo parcial un $2 \%$, lo cual muestra, además, que la mayor parte de docentes trabajan a tiempo completo.

En el caso de la U1, la edad de los docentes no difiere de la media general de estudio. El $46 \%$ de docentes tiene edad entre 36 y 50 años, el $74 \%$ pertenece al género masculino, el $65 \%$ cuenta con maestría y en su mayoría tienen un tiempo de servicio en la universidad entre 1 y 10 años. La mayoría de los docentes trabaja a tiempo completo y tiene nombramiento, y solamente un $7 \%$ trabaja a medio tiempo o a tiempo parcial.

Los docentes de la U2, en un $43 \%$ tienen edades entre 36 y 50 años, un $70 \%$ pertenece al género masculino y un $67 \%$ cuenta con formación de maestría. Además, el $46 \%$ de docentes tiene entre 1 y 10 años de servicio en la universidad y todos dijeron trabajar a tiempo completo. De ellos, un $97 \%$ tiene nombramiento.

El $78 \%$ de los docentes de la U3 tiene entre 36 y 50 años, el $67 \%$ pertenece al género masculino, el $84 \%$ cuenta con formación de magister, el resto corresponde a PhD. En cuanto al tiempo de servicio, un $50 \%$ tiene entre 1 y 10 años, y el $32 \%$ de 11 a 20 años. El $95 \%$ tienen nombramiento y $76 \%$ de ellos trabaja a tiempo completo.

\section{Análisis de la cultura organizacional}

\section{Integración}

El promedio total de la U1 es 2.61, entendiéndose que 5 es totalmente de acuerdo, por tanto, este valor implica un bajo nivel de integración o indica una cierta indiferencia en aspectos asociados a la innovación, cumplimiento de objetivos, salario, ambiente de cooperación, entre otras.

El promedio de la U2 es 2.82, es aún bajo en los diferentes aspectos evaluados, por tanto, existen varios aspectos que no están fortalecidos en la universidad, por tanto, no genera una cultura de integración en los docentes.

La U3 obtuvo un promedio total de 3.75 , lo cual indica que, si bien existe una variabilidad en las respuestas, el valor es medianamente alto; es decir que se ubica entre una posición media cercana a que están de acuerdo en la mayor parte de los puntos analizados. De las tres universidades, esta obtuvo una puntuación bastante elevada en contraste con las otras.

\section{Relaciones interpersonales}

El promedio total de los ámbitos de relaciones personales para la U1 es 2.8, lo cual indica que, aunque en algunos ámbitos de esta variable está un porcentaje importante de los docentes de acuerdo; en otros ámbitos están en su mayoría en desacuerdo, por tanto, ha obtenido un resultado intermedio, algo indiferente o en desacuerdo en general en que existen adecuadas relaciones interpersonales.

En el caso de la U2 el promedio es 2.82, igual al caso anterior, es decir, una percepción indiferente o algo en desacuerdo acerca de que existen adecuadas relaciones interpersonales.

En la U3 las relaciones interpersonales son superiores a las otras dos, con un valor promedio final de 3.84 , lo cual indica que, con- 
siderando ámbitos como las relaciones entre miembros, el buen trato, el reconocimiento y las relaciones entre áreas están de acuerdo en que existen buenas relaciones interpersonales.

\section{Trabajo en equipo}

El promedio para la U1 es 2.63, lo cual muestra un valor bajo y una percepción indiferente 0 algo en desacuerdo en que existe una cultura adecuada en relación con el trabajo en equipo. En la $\cup 2$ el promedio en equipo medio es 2.68, similar al de la U1. En la U3, el trabajo en equipo puntúa en promedio 3.66 , un valor bastante superior a las otras dos universidades, por lo que se entiende que están de acuerdo con que existe trabajo en equipo, coordinación entre áreas, se favorece a las innovaciones y soluciones de conflictos.

\section{Comunicación}

En relación con la comunicación, la U1 obtuvo un promedio general 3.2, valor que implica que existen algunas variables en las que la comunicación existe, pues un porcentaje importante de los docentes están de acuerdo en ello. El promedio de la U2 es 3.10, por tanto, la comunicación también es aceptable. Finalmente, la comunicación en la U3 es algo alta, es decir que sí existe comunicación y, en su mayoría, los docentes están de acuerdo, lo que se evidencia mediante la comunicación entre compañeros, en la retroalimentación de los jefes e información compartida.

\section{Desempeño docente}

\section{Contenidos}

Mediante la autoevaluación de los docentes de estas universidades se obtuvo los siguientes resultados:

El puntaje promedio total para la U1 de la variable contenido es de 4.07. Esto indica, respaldando el análisis previo, que mayormente en relación con la valoración de contenido los docentes están de acuerdo en cumplir con los diferentes parámetros evaluados.

Para la U2, similar al caso de la U1, la mayor parte de preguntas son contestadas con 'de acuerdo' (65\%), tanto en la "diversificación de contenidos tomando en cuenta la realidad" como en "elaborar las unidades de aprendizaje de acuerdo al contexto" (54\%), uso de las metodologías y diversificación. Sin embargo, en este grupo de maestros un porcentaje importante se muestra indiferente ante estas afirmaciones, lo cual causa que su promedio de puntuación total disminuya a 3.69. Esto podría indicar que su respuesta está entre 'de acuerdo' y 'ni de acuerdo' / 'ni en desacuerdo' o 'indiferente', lo que muestra un menor desempeño en comparación con el caso anterior.

Al analizar los resultados de la $U 3$, se observa un comportamiento diferente a los casos anteriores. En esta universidad, la mayor parte de los docentes está completamente de acuerdo (64\%) en las aseveraciones acerca de que "realiza diversificación de contenidos tomando en cuenta la realidad", así como en "elaborar las unidades de aprendizaje de acuerdo al contexto" (57\%), uso de las metodologías y diversificación. La universidad obtuvo promedios de 4.41 para la primera pregunta y el mínimo valor es de 4.11, lo cual lleva a un resultado promedio de 4.3 -superior a los casos anteriores- que muestra que el desempeño medido con base en la variable contenidos es mayor que en los otros casos. Si bien este resultado corresponde a su autoevaluación, muestra resultados claramente distintos a los casos anteriores.

\section{Planificación}

Con relación a la variable planificación, las preguntas de análisis están en torno a si los objetivos guardan relación con la visión y mi- 
sión de la institución, si se elabora la planificación del área, si se logran los objetivos que se proponen, si los resultados son provechosos y si los recursos utilizados en clase son adecuados para presentar la realidad.

Los docentes de la U1 están de acuerdo con la mayor parte de respuestas, con valores medios entre 3.78 y 4.21 , esto quiere decir que para los docentes las actividades con el alumnado al terminar cada proceso de enseñanza son provechosos. Sí existe una planificación y, para la mayoría, se alcanzan los objetivos propuestos. No obstante, un menor porcentaje está de acuerdo en que los recursos utilizados son idóneos o que los objetivos están relacionados con la visión y misión de la institución. El promedio obtenido fue 3.98, cercano a 4, al valorar la planificación en el desempeño docente.

La U2 muestra valores algo menores comparados con la U1. Esta universidad obtuvo resultados 'de acuerdo' de la mayor parte de docentes, pero algunos tienen una posición indiferente, con lo cual los promedios que verifican la relación entre los objetivos y la visión y misión de la institución tienen una media de 3.35, por tanto, para muchos, no se cumple en su totalidad esta relación. Asimismo, consideran que los recursos utilizados son adecuados para representar la realidad, pero sí están mayormente de acuerdo en que las actividades del alumnado al terminar el proceso son provechosas. La media total de la variable planificación para la U2 es de 3.7, lo cual indica una posición media entre indiferente y de acuerdo.

En cuanto a la U3, los resultados de planificación se muestran favorables. La mayor parte de los docentes $(60 \%)$ cree que los objetivos sí guardan relación con la misión y visión de la institución; además, aseguran que se elabora una planificación de área, se logran los objetivos propuestos, y sí consideran provechosas las actividades al terminar cada proceso y, finalmente, con- sideran que los recursos utilizados en el aula son adecuados. El promedio general es 4.32.

\section{Evaluación}

El promedio para la variable evaluar es de 3.42 para la U1. Los demás valores son algo bajos para la U2, que alcanzó un promedio total de 2.84, lo cual indicaría que la media considera entre indiferente y estar de acuerdo; es decir que puntúan la evaluación con un valor bajo. Para la U3, la evaluación muestra valores más altos comparados con la U1 y U2, pero también las dos preguntas han generado que los docentes se manifiesten un porcentaje en contra de la metodología para supervisión, así como la evaluación de desempeño. Las demás variables obtuvieron puntajes altos, con un promedio total de 3.98 que refleja un puntaje algo alto para la variable evaluación del desempeño docente.

\section{Desempeño general}

Finalmente, el desempeño general con base en las preguntas relacionadas con la organización del trabajo, su metodología, conocimientos, uso de recursos, prácticas, estrategias, aplicación de principios, investigación, hace uso de una entrega, puntualidad, atención al perfil del estudiante, satisfacción, participación, entre las principales. Los resultados son los siguientes:

El promedio para la U1 en relación con el desempeño general es 4.29, es decir, una gran mayoría está de acuerdo en que existe un buen desempeño como docente, de acuerdo con su evaluación.

El promedio para el desempeño general en la U2 es 4.03, un valor alto que implica que están de acuerdo y muchos completamente de acuerdo en las diferentes variables analizadas, con lo cual muestran un buen desempeño con base en su propia evaluación. 
Para la U3, en la mayor parte de variables el desempeño general es alto. Los docentes están completamente de acuerdo en los diversos temas de respeto, puntualidad, entrega, servicio y vocación al trabajo, participación activa y, de ello, el tener una alta sa- tisfacción en su labor docente. El promedio obtenido en los diferentes aspectos de desempeño general es de 4.52, es decir, están de acuerdo y completamente de acuerdo en la mayor parte de aspectos analizados como variables del desempeño general (tabla 4).

Tabla 4. Desempeño docente

\begin{tabular}{|l|c|c|c|c|}
\hline \multirow{2}{*}{ Universidades } & \multicolumn{4}{|c|}{ Componentes del desempeño } \\
\cline { 2 - 5 } & Contenidos & Planificación & Evaluación & Desempeño docente \\
\hline U1 & 4.07 & 3.98 & 3.42 & 4.29 \\
\hline U2 & 3.69 & 3.70 & 2.84 & 4.03 \\
\hline U3 & 4.30 & 4.32 & 3.98 & 4.52 \\
\hline
\end{tabular}

\section{Desempeño docente medido, de acuerdo con la evaluación realizada por las universidades}

Se han obtenido los resultados de la evaluación de desempeño aplicados en las uni- versidades, y regulados por el UAACES. De estos el resultado cuantitativo de la evaluación media, de acuerdo con las variables de docencia, investigación y vinculación es el siguiente (tabla 5):

Tabla 5. Resultados de evaluación

\begin{tabular}{|l|c|c|c|c|}
\hline Universidades & Docencia & Investigación & Vinculación & Media \\
\hline U1 & 8.34 & 8.83 & 8.67 & 8.61 \\
\hline U2 & 8.45 & 9.2 & 8.9 & 8.85 \\
\hline U3 & 9.4 & 8.8 & 9.51 & 9.24 \\
\hline
\end{tabular}

Los resultados numéricos de las evaluaciones medidas por las universidades se puntuaron con una calificación sobre 10 puntos. Los informes presentados muestran muy poca información cualitativa de acuerdo con el tipo de estudio; sin embargo, los resultados numéricos finales presentan resultados de valor con los cuales se puede hacer un primer acercamiento correlacional entre las variables cultura y desempeño docente.

Se puede constatar, en general, valores altos entre 8.34 y 9.51, de acuerdo con la puntuación de cada caso. La U3 tiene los valores más altos, con 9.24 de promedio, mientras los menores corresponden a la U1 con
8.61. Las menores calificaciones en cuanto a docencia y vinculación corresponden a la U1 con 8.34 y 8.67 respectivamente. La U3 obtuvo valores mayores en docencia y vinculación. Con estos valores fue posible hacer un análisis correlacional entre variables.

\section{Relación entre la cultura y el desempeño}

Un primer acercamiento a los resultados se puede obtener al comparar los resultados de cultura, medida a través de las variables de integración, relaciones interpersonales, trabajo en equipo y comunicación, con el 
desempeño, medido con variables como contenidos, planificación, evaluación y des-

empeño general. En la tabla 6 se presentan estos resultados:

Tabla 6. Relación entre cultura y el desempeño

\begin{tabular}{|c|c|c|c|c|c|c|c|c|}
\hline \multirow{2}{*}{ U } & \multicolumn{4}{|c|}{ Cultura } & \multicolumn{4}{c|}{ Desempeño } \\
\cline { 2 - 9 } & Integración & $\begin{array}{c}\text { Relaciones } \\
\text { interperso- } \\
\text { nales }\end{array}$ & $\begin{array}{c}\text { Trabajo } \\
\text { en equipo }\end{array}$ & $\begin{array}{c}\text { Comuni- } \\
\text { cación }\end{array}$ & $\begin{array}{c}\text { Conteni- } \\
\text { dos }\end{array}$ & $\begin{array}{c}\text { Planifica- } \\
\text { ción }\end{array}$ & Evaluación & $\begin{array}{c}\text { Desempe- } \\
\text { ño general }\end{array}$ \\
\hline U1 & 2.61 & 2.80 & 2.63 & 3.20 & 4.07 & 3.98 & 3.42 & 4.29 \\
\hline U2 & 2.82 & 2.82 & 2.68 & 3.10 & 3.69 & 3.70 & 2.84 & 4.03 \\
\hline U3 & 3.75 & 3.84 & 3.66 & 3.78 & 4.30 & 4.32 & 3.98 & 4.52 \\
\hline
\end{tabular}

Una sumatoria simple de los resultados permitió obtener una calificación ge- neral tanto de cultura como de desempeño (tabla 7).

Tabla 7. Resumen de Cultura y desempeño

\begin{tabular}{|l|c|c|}
\hline Universidades & Total Cultura & Desempeño \\
\hline U1 & 11.24 & 15.75 \\
\hline U2 & 11.41 & 14.26 \\
\hline U3 & 15.02 & 17.12 \\
\hline
\end{tabular}

Al analizar la correlación entre las variables total cultura y desempeño, se obtuvo el gráfico de figura 1.

Figura l. Relación cultura y desempeño

\section{Desempeño}

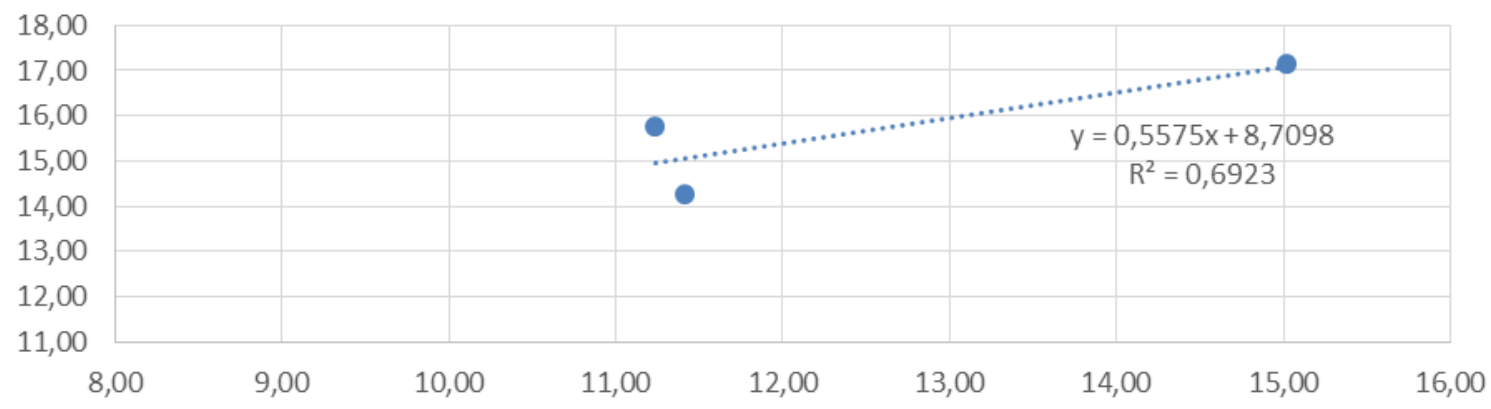

En el gráfico se puede ver una tendencia lineal clara; en la que: a mayor calificación de cultura, mayor calificación en desempeño existe. El coeficiente de correlación es bastante alto, con un valor $r$ de $0.83 \mathrm{y} \mathrm{r}^{2}$ de 0.69 , lo cual implica una correlación fuer- te entre desempeño y cultura. Sin embargo, es importante recordar que la muestra es pequeña, porque está conformada por las tres universidades públicas del Ecuador que se encuentran en las categorías A y B. Además de que el análisis fue elaborado to- 
mando en cuenta la autoevaluación de desempeño, por lo que es fundamental hacer el mismo análisis con los datos de desempeño medidos a través de las universidades internamente.
Para verificar la correlación, se hizo una prueba de hipótesis para los datos obtenidos de cultura y desempeño medio. Dado que la cantidad de datos es pequeña, se utiliza el análisis de correlación no paramétrico de Spearman y Kendall (tabla 8).

Tabla 8. Correlaciones

\begin{tabular}{|c|c|c|c|c|}
\hline & & & Cultura & Desempeño \\
\hline \multirow{6}{*}{ Tau_b de Kendall } & \multirow{3}{*}{ Cultura } & Coeficiente de correlación & 1.000 & 1.000 \\
\hline & & Sig. (bilateral) & . & . \\
\hline & & $\mathrm{N}$ & 3 & 3 \\
\hline & \multirow{3}{*}{ Desempeño } & Coeficiente de correlación & $1.000^{* *}$ & 1.000 \\
\hline & & Sig. (bilateral) & . & . \\
\hline & & $\mathrm{N}$ & 3 & 3 \\
\hline \multirow{6}{*}{$\begin{array}{c}\text { Rho de } \\
\text { Spearman }\end{array}$} & \multirow{3}{*}{ Cultura } & Coeficiente de correlación & 1.000 & $1.000^{* *}$ \\
\hline & & Sig. (bilateral) & . & . \\
\hline & & $\mathrm{N}$ & 3 & 3 \\
\hline & \multirow{3}{*}{ Desempeño } & Coeficiente de correlación & $1.000^{\star *}$ & 1.000 \\
\hline & & Sig. (bilateral) & . & . \\
\hline & & $\mathrm{N}$ & 3 & 3 \\
\hline
\end{tabular}

Nota: **La correlación es significativa en el nivel 0.01 (bilateral)

La correlación es significativa para un $99 \%$ de confiabilidad, lo cual corrobora la relación.
Asimismo, se analizó las correlaciones entre las diferentes variables de cultura, así como de desempeño y se obtuvo diferentes coeficientes de correlación (tabla 9).

Tabla 9. Coeficientes de correlaciones

\begin{tabular}{|l|c|c|c|c|}
\hline & Integración & $\begin{array}{c}\text { Relaciones } \\
\text { interpersonales }\end{array}$ & $\begin{array}{c}\text { Trabajo en } \\
\text { equipo }\end{array}$ & Comunicación \\
\hline Contenidos & 0.67 & 0.77 & 0.76 & 0.87 \\
\hline Planificación & 0.81 & 0.89 & 0.88 & 0.95 \\
\hline Evaluación & 0.76 & 0.85 & 0.84 & 0.93 \\
\hline & 0.75 & 0.84 & 0.83 & 0.92 \\
\hline
\end{tabular}

La mayor parte de variables de cultura tiene altos niveles de correlación con las variables de desempeño, lo cual implica que el desempeño sí depende de la cultura de la institución universitaria. El menor valor, (0.67), se presenta en la relación entre desempeñocontenidos y cultura-integración, lo cual implica que el desempeño de los docentes en relación con los contenidos no tiene una relación muy estrecha con la integración. 


\section{Comparación cuantitativa de los valores de cultura y desempeño}

Tomando en cuenta el desempeño medido por las universidades mediante evaluación interna, comparativamente, según las calificaciones obtenidas, se obtuvo lo siguiente (tabla 10):

Tabla 10. Cultura total y desempeño

\begin{tabular}{|l|c|c|}
\hline Universidades & Total Cultura & Desempeño \\
\hline U1 & 11.24 & 8.61 \\
\hline U2 & 11.41 & 8.85 \\
\hline U3 & 15.02 & 9.24 \\
\hline
\end{tabular}

Los valores no presentan diferencias importantes en U2 y U1, pero U3 muestra una brecha mayor entre la cultura y desempeño, los cuales fueron medidos en las evaluaciones de las universidades y los resultados de cultura evaluados por los docentes. Al analizar la correlación entre las variables total cultura y desempeño, se obtuvo el siguiente gráfico (figura 2)

Figura 2. Relación cultura y desempeño

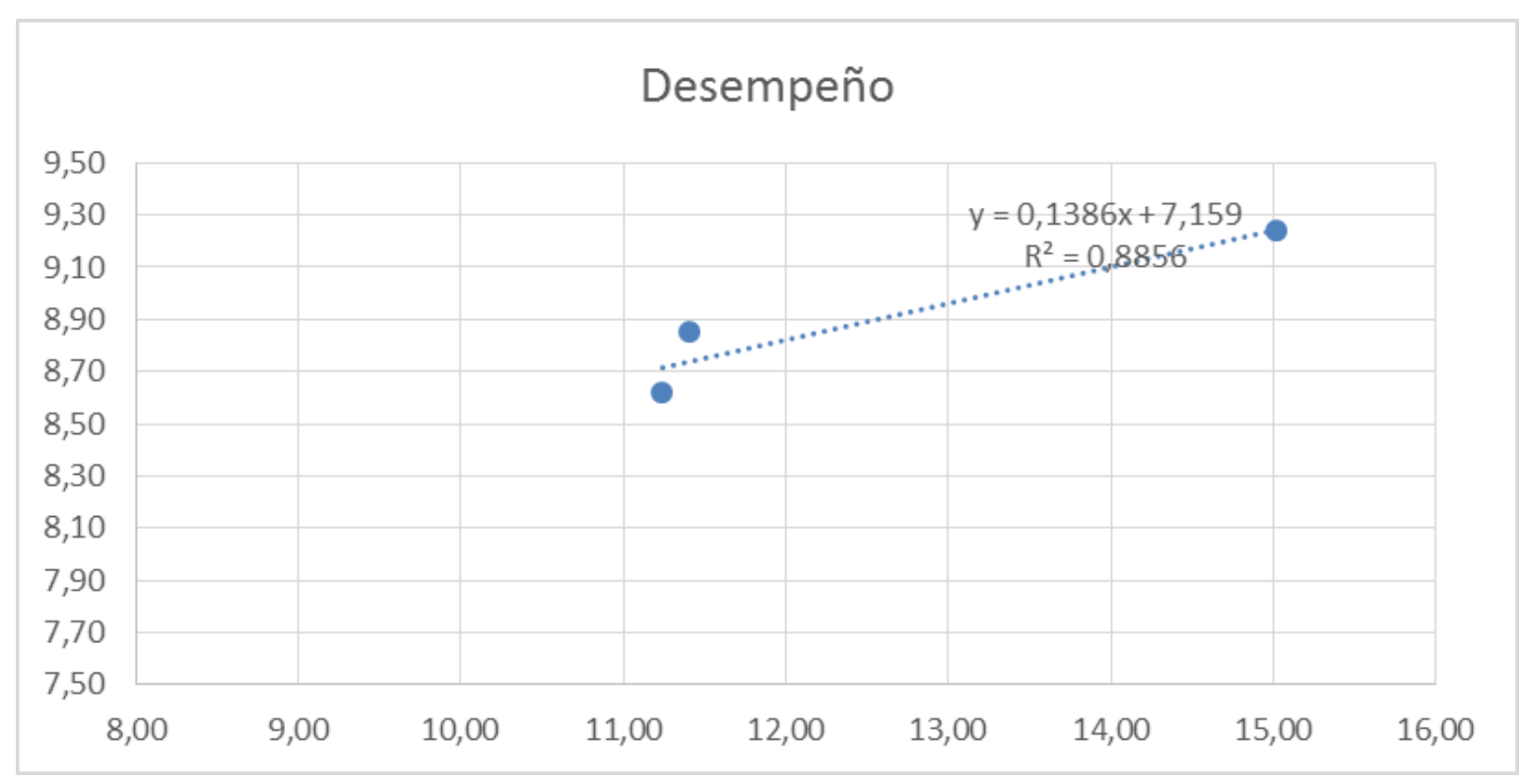

El valor de r para esta evaluación es de 0.94, por lo tanto, sí existe una correlación; sin embargo, este resultado muestra aún mayor correlación que la valoración hecha mediante la autoevaluación y se pudieron verificar los resultados previos, donde la universidad que tiene menor puntaje en cultura también tiene un menor valor de desempeño; así también, la que mayor tiene puntaje en cultura, también lo tiene en desempeño medido por la propia universidad. Los resultados son muy similares en ambos casos, pero con la variación presentada.

A continuación, se analizan las variaciones presentadas por las variables de estudio, donde el desempeño, finalmente, es similar en ambos casos (tabla 11). 
Tabla 11. Resultados de las variables

\begin{tabular}{|c|c|c|c|c|c|c|c|}
\hline \multicolumn{3}{|c|}{ Cultura } & \multicolumn{3}{c|}{ Desempeño } \\
\hline & $\begin{array}{c}\text { Integra- } \\
\text { ción }\end{array}$ & $\begin{array}{c}\text { Relaciones } \\
\text { interpersonales }\end{array}$ & $\begin{array}{c}\text { Trabajo en } \\
\text { equipo }\end{array}$ & $\begin{array}{c}\text { Comunica- } \\
\text { ción }\end{array}$ & Docencia & $\begin{array}{c}\text { Investiga- } \\
\text { ción }\end{array}$ & Vinculación \\
\hline U1 & 2.61 & 2.80 & 2.63 & 3.20 & 8.34 & 8.83 & 8.67 \\
\hline U2 & 2.82 & 2.82 & 2.68 & 3.10 & 8.45 & 9.20 & 8.90 \\
\hline U3 & 3.75 & 3.84 & 3.66 & 3.78 & 9.40 & 8.80 & 9.51 \\
\hline
\end{tabular}

Finalmente, al analizar las correlaciones entre cultura y desempeño, se puede ver que las correlaciones entre docencia y relaciones interpersonales y trabajo en equipo y comunicación son muy altas; es decir, las variables de desempeño de docencia están altamente correlacionadas con las de cultura organizacional, lo que muestra que una mejor cultura ayuda a fortalecer el desempeño medido de acuerdo con la variable docencia. Aparte, existe una correlación negativa y con menor fuerza entre las variables de cultura y la investigación. El motivo no es claro, pero puede deberse a factores como el hecho de que la investigación es una variable que aún no está bien desarrollada en el Ecuador y, en ciertos casos, la investigación es una obligación en las universidades públicas, por lo que no se muestra correlación entre investigación y cultura organizacional. Respecto a la variable vinculación y docencia, sí existe una correlación con las variables de cultura, lo cual demuestra, en general, que la cultura sí depende de la vinculación (tabla 12).

Tabla 12. Correlación de las variables de cultura y desempeño de acuerdo con el análisis de las variables

\begin{tabular}{|l|c|c|c|}
\hline \multicolumn{1}{|c|}{ Variables de cultura } & Docencia & Investigación & Vinculación \\
\hline Integración & 1.00 & -0.41 & 1.00 \\
\hline Relaciones interpersonales & 1.00 & -0.54 & 0.97 \\
\hline Trabajo en equipo & 1.00 & -0.53 & 0.97 \\
\hline Comunicación & 0.97 & -0.67 & 0.92 \\
\hline
\end{tabular}

\section{Discusión y conclusiones}

En la U1, desde el ámbito directivo, la cultura está concebida como algo complicado, no está claramente definida, es muy variada y, en ciertos casos, falta desarrollar compromiso. No hay suficiente motivación ni comunicación, además de que los docentes no se identifican con la entidad.

Respecto del desempeño, aunque hay investigación, se cumple con los estándares establecidos por la institución y existen ámbitos favorables, es claro que en relación con la docencia falta capacitación. Finalmente, en el ámbito vinculación, la U1 tiene fortalezas.

En la U2, en relación con la cultura, existen aspectos positivos como la relación interpersonal y la integración, pero también hay grupos que discrepan en su pensamiento; además de que el trabajo en equipo, en general, no es la mayor fortaleza.

En cuanto al desempeño, están motivados por la investigación y cuentan con procedimientos y presupuesto para ella; sin embargo, las actividades de investigación no se valoran lo suficiente. Respecto a otros facto- 
res de la docencia, existen ámbitos favorables como el profesionalismo de sus docentes, el apego a los valores y el compromiso con el estudiante. En contraste, consideran que faltan recursos e infraestructura necesaria para sus requerimientos. Por último, internamente no se percibe la importancia de la vinculación.

En el caso de la U3, en opinión de los directivos, existe un trabajo participativo entre los docentes, aunque también se menciona que este es difícil, debido al tamaño de la institución. Se organiza la elaboración de programas y existe muy buena comunicación y compromiso. Su cultura sí está bien definida y los docentes se identifican con la universidad.

En cuanto a la evaluación docente, se puede decir que la investigación no es uno de los fuertes de la institución, pero se está mejorando; sin embargo, en relación con la docencia existen muy buenos resultados, aunque están conscientes de que aún deben mejorar. Por último, la vinculación es un requisito en el que están integrados los docentes.

Finalmente, al consultarles a los docentes acerca de los aspectos que consideran que se deberían mejorar en la cultura organizacional, mencionaron los siguientes:

- Para impulsar una cultura organizacional abierta, positiva, agradable, que potencie la actividad del docente, se requiere de un esfuerzo y trabajo continuo. Se debe definir una estrategia con objetivos claros, acompañada de reglamentación y procedimientos precisos. Además, es necesario retroalimentar al docente después de las evaluaciones, generar ambientes de confianza y hablar tanto de lo negativo como de los éxitos y triunfos.

- Es importante socializar en la universidad, además de tener los recursos y la estabilidad en la dirigencia para poder obtener resultados.

- En la universidad pública, el ámbito político y la falta de estabilidad tienen efectos sobre la cultura y, por tanto, es un punto que se debe internalizar para corregir o mejorar.

La administración, de acuerdo con sus principales exponentes, establece la cultura organizacional, entre otras variables relacionadas con la satisfacción laboral, como un aspecto que está vinculado con el desempeño del personal. Consecuentemente, cuando existe una mayor satisfacción en el trabajo, se asume una cultura más fuerte y favorable para el personal, el desempeño es mejor y los resultados de las entidades también, pues del personal y su desempeño depende el éxito de las organizaciones.

Esta es la base que justifica este análisis por medio del que se pretende conocer si, a nivel de las universidades públicas en el Ecuador, la cultura genera efectos sobre el desempeño docente, pues usualmente en estas instituciones el enfoque está más orientado al desarrollo intelectual, la investigación, la metodología, la pedagogía. No obstante, el ámbito organizacional es básico para analizar y determinar su incidencia en el desempeño y, a su vez, para entender que de este dependen los resultados de estudiantes bien formados, con el perfil establecido y valores y competencias del más alto nivel, debido a que la formación estudiantil siempre estará relacionada con la educación ofrecida por los docentes.

En la investigación se consideró una muestra pequeña de universidades, aun así, se ha podido demostrar estadísticamente que la cultura organizacional en las universidades públicas tiene efecto sobre el desempeño de los docentes. Debido al carácter exploratorio de la investigación fue posible hacer un primer acercamiento a la relación entre cultura organizacional y desempeño docente considerando la importancia de este desempeño en la efectividad de los resultados de la universidad, y la necesidad de analizar cómo se relacionan estas dos variables. 
Los resultados muestran que dos de las universidades cuentan con una cultura organizacional más desarrollada en cuanto a integración, relaciones interpersonales, trabajo en equipo y comunicación. La puntuación dada en promedio por parte de los docentes para las universidades U1 y U2 fue de 2.6 hasta 3.2 puntos, lo que refleja que no están bien fundamentados muchos de los factores de la cultura organizacional que han sido medidos; aunque existe variabilidad en los mismos de acuerdo con los diferentes encuestados, la media muestra que no existe un alto nivel de satisfacción en cuanto a la cultura existente. Los resultados de la U3, cuyos valores promedio sobre 5 van desde 3.66 hasta 8.84 , fueron excepcionales. Sus valores fueron mucho mayores y reflejan, al contrario que las otras dos instituciones, que en su mayoría los docentes están de acuerdo en que se manifiestan la mayor parte de las variables de cultura estudiadas. Estos resultados muestran que existen diferencias significativas entre la cultura organizacional en una universidad en relación con las otras, de lo cual se parte para analizar si estos niveles de cultura organizacional inciden en el desempeño de los docentes de las respectivas instituciones.

Analizando la variable desempeño docente, en el estudio se ha considerado, por una parte, la evaluación de desempeño que aplica cada entidad universitaria, mediante metodologías apegadas a la normativa nacional y, por otra, la autoevaluación aplicada a los docentes, en la cual se midió contenidos, planificación, evaluación, entre otros factores de desempeño docente. Ambas evaluaciones llevaron a conclusiones similares. Es decir, las U1 y $U 2$ obtuvieron resultados cuantitativos medios, y la U3, resultados altos. Al hablar de altos, de acuerdo con la autoevaluación, se refiere a que, en su mayoría, los docentes están de acuerdo con que cada uno se desempeña adecuadamente en lo relativo a planificación, evaluación y contenidos; a su vez, cuando las universidades miden el desempeño, se refiere a docencia, investigación y vinculación. En ambos casos los resultados fueron similares para cada universidad, la U3 obtuvo mejor puntuación general a diferencia de U1 y U2. Estos resultados suponen una primera visión de que sí existe una correlación entre cultura y desempeño docente.

El análisis de correlación desarrollado permitió demostrar con un $99 \%$ de confiabilidad. Este fue medido mediante pruebas no paramétricas de correlación (Rho de Spearman y el Tau_b de Kendall), las cuales entregaron valores muy cercanos a 1 y demostraron una correlación significativa entre los valores medios de cultura organizacional y desempeño docente. En consecuencia, se pudo se pudo verificar la hipótesis acerca de que las variables de la cultura organizacional, es decir, comunicación, trabajo en equipo, relaciones interpersonales e integración sí inciden en el desempeño de los docentes universitarios, en sus funciones de docencia, investigación y vinculación, de las universidades públicas de la provincia de Pichincha.

Se ha podido detectar, aunque no en todos los casos, varias debilidades e insuficiencias de los factores de la cultura organizacional que podrían dificultar alcanzar las metas institucionales. Sobre este tema se podrían profundizar en futuras investigaciones, abordando aspectos que necesitan intervención y los procesos para ajustarlos y eliminar sus efectos, con el objeto de mejorar la calidad educativa y las metas institucionales y nacionales en cuanto a educación superior.

\section{Referencias}

Anzola, M.O. (2003). Una mirada de la cultura corporativa. Editorial Universidad externado de Colombia.

Arias, F. (2012). El proyecto de investigación: Introducción a la metodología científica. (6. ${ }^{a}$ Ed.). Episteme. 
Benítez Torres, D. (2007, agosto 28). Efectividad de la evaluación del desempeño docente en una universidad. Gestiopolis. https:// bit.ly/3EbeUeh

Cheung, S., Wong, P., \& Lam, A. (2012). An investigation of the relationship between organizational culture and the performance of construction organizations. Journal of Business Economics and Management, 13(4), 688-704.

Consejo de Aseguramiento de la Calidad de la Educación Superior (CACES). (2018). Categorías de universidades del Ecuador SENESCYT. CACES

Consejo de Aseguramiento de la Calidad de la Educación Superior (CACES). (2019). Modelo de evaluacion. CACES.

Daft, R. L. (2011). Teoría y diseño organizacional (10. ${ }^{a}$ Ed.). Cengage Learning.

De Aparicio, X., Chininin, M., \& Toledo, O. (2017). El rol de la vinculación en la integración de las funciones sustantivas de la Universidad Metropolitana del Ecuador. Universidad y Sociedad, 9(4), 37-43. https://bit. ly/3CbEdfB

de Hilal, A.V.G., Wetzel, U., \& Ferreira, V. (2009). Organizational culture and performance: a Brazilian case. Management Research News, 32, 99-119

Escuela Politécnica del Ejército (ESPE). (2019). Población docente. Unidad de Talento Humano ESPE.

Escuela Politécnica Nacional (SII-EPN). (2018). Listado de número de profesores por departamento: Periodo 2018. SII-EPN.

Figueroa, L. (2016). Relación entre la cultura organizacional y el desempeño laboral de los colaboradores de una institución gubernamental. [Tesis de grado, Universidad Rafael Landivar]. Tesario de la Red de Bibliotecas Landivarianas. https://bit. ly/3w9JJx6

Garcés, L. M. (2013). Teorías tradicionales de la administración: La cultura organizacional desde una perspectiva crítica. [Tesis de grado, Universidad Pontificia Bolivariana]. https://bit.ly/2ZLtJ92

Gómez López, L.F., \& Valdés Dávila, M.G. (2019). La evaluación del desempeño docente en la educación superior. Propósitos y Representaciones, 7(2), 479-515. http://dx.doi. org/10.20511/pyr2019.v7n2.255

Góngora, N., Nóbile, C., \& Reija, L. (2014). Estudio comparativo de la cultura organizacional. Ciencias Administrativas, 2(4), 51-65. https://bit.ly/3qklw5e

González-Such, J., \& Subaldo-Suizo, L. (2015). Opiniones sobre el desempeño docente y sus repercusiones en la satisfacción profesionaly personal de los profesores. Educación, 24(47), 90-114. https://bit.ly/3oh2dzf

Greenbaum, H. (2017). The audit of organizational communication. Academy of Management Proceedings, 1974(1). https://doi. org/10.5465/ambpp.1974.17531485

Hernández, R., Fernández, C., \& Baptista, P. (2014). Metodología de la investigación. (6ta Ed.). McGraw-Hill; Interamericana.

Linares, I., Ochoa, S., \& Ochoa-Silva, B. (2013). Cultura organizacional y evaluación del desempeño del personal académico: Estudio de caso en una institución de educación superior pública mexicana. Revista Electrónica Nova Scientia, 6(11), 324-345. http://doi.org/10.21640/ns.v6i11.86

Loredo, J. (2015). Examinar la evaluación de la docencia: Un ejercicio imprescindible de investigación institucional. Revista de la Educación Superior, 44(174), 157-166. http://doi.org/10.1016/j.resu.2015.06.004

Loren, M. (2012, febrero 27). La cultura organizacional. Escuela de Organización Industrial https://bit.ly/3b6tpni

Ludlow, R., \& Panton, F. (1997). La esencia de la comunicación. Prentice-Hall.

Martínez Ruiz, S. I., \& Lavín García, J. L. (2017, abril 5). Aproximación al concepto de desempeño docente: Una revisión conceptual sobre su delimitación. [Ponencia]. XIV Congreso Nacional de Investigación Educativa, San Luis Potosí, México. https:// bit.ly/3mdL2YF

Mascaray, J. (1998). Más allá de la comunicación interna. La intercomunicación: Diez estrategias para la implementación de valores y la conquista del comportamiento espontáneo de los empleados. Gestión 2000. 
Montes Padilla, F.G. (2016, marzo 9). Relaciones interpersonales en el trabajo. Visión Industrial. https://bit.ly/3vJw6Va

Montesinos Flores, A. (2014). Maestro, académico universitario y el rumbo adecuado en el ejercicio. Revista Mexicana de Ortodoncia, 2(1), 6-8. https://bit.ly/3nwyA5s

Moreno Bastidas, G.R. (2020). La cultura organizacional en el desempeño docente en las universidades públicas de la provincia Pichincha, Ecuador. [Tesis de Doctorado, Universidad Nacional de La Plata]. Repositorio Institucional.

Murraina, E., Farid Barrera, N., \& Vargas, Y. (2017). Cuatro reflexiones sobre la docencia. Repertorio de Medicina y Cirugía, 26(4), 242-248. https://doi.org/10.1016/j.reper.2017.09.001

Reinoso, H., \& Araneda, B. (2007). Diseño y validación de un modelo de medición de clima organizacional basado en percepciones y expectativas. Revista Ingeniería Industrial, 39-54. https://bit.ly/2ZL8Rid

Rivera, D., Carrillo, S., Forgiony, J., Nuván, I., \& Rozo, A. (2018). Cultura organizacional, retos y desafíos para las organizaciones saludables. Revista Espacios, 39(22), 27-41. https://bit.ly/3CHD2oq

Salazar Estrada, J.G., Guerrero Pupo, J.C., Machado Rodríguez, Y.B., \& Cañedo Andalia, R.
(2009). Clima y cultura organizacional: dos componentes esenciales en la productividad laboral. Acimed, 20(4), 67-75.

Sampieri, R.H., Valencia, S.M., \& Soto, R.C. (2014). Construcción de un instrumento para medir el clima organizacional en función del modelo de los valores en competencia. Contaduría y administración, 59(1), 229-257.

Sánchez, J., \& Otálora, J. (2006). Fundamentos de trabajo en equipo para equipos de de trabajo. McGraw-Hill.

Segredo Pérez, A. M. (2016). Desarrollo organizacional: Una mirada desde el ámbito académico. Educación Médica, 17(1), 3-8. https:// doi.org/10.1016/j.edumed.2015.09.002

Schein, E.H. (2004). Organizational culture and leadership. 3ra ed. John Wiley \& Sons, Inc.

Sistema Nacional de Evaluación, Acreditación y Certificación de la Calidad Educativa (Sineace). (2017). Experiencias de investigación en institutos de educación superior pedagógicos. Sineace.

Valdiviezo, C., Valdivieso, R., \& Valdivieso, 0. (2011). Determinación del tamaño muestral mediante el uso de árboles de decisión. Investigación \& Desarrollo, 11, 148176. https://bit.ly/3CFF4Wh 